\title{
A simple theory of lags in gamma-ray bursts: Comparison to observations
}

\author{
R. Mochkovitch ${ }^{1}$, V. Heussaff ${ }^{2,3}$, J. L. Atteia ${ }^{2,3}$, S. Boçi ${ }^{4}$, and M. Hafizi ${ }^{4}$ \\ 1 Sorbonne Universités, UPMC Université Paris 6 et CNRS, UMR 7095, Institut d'Astrophysique de Paris, 75014 Paris, France \\ e-mail: mochko@iap.fr \\ 2 Université de Toulouse, UPS-OMP, IRAP, 31400 Toulouse, France \\ 3 CNRS IRAP, 14 avenue Édouard Belin, 31400 Toulouse, France \\ 4 Tirana University, Faculty of Natural Sciences, Tirana, Albania
}

Received 20 October 2015 / Accepted 25 April 2016

\begin{abstract}
Context. Lags observed between the light curves of a gamma-ray burst (GRB) seen in different energy bands are related to its spectral evolution. Moreover the lags have been found to correlate with burst luminosity, therefore providing a potential distance indicator. Aims. We want to quantify the nature of the link between lags and spectral evolution to better understand the origin of the lagluminosity relation and evaluate its interest as a distance indicator.

Methods. We directly relate the lag of a pulse to the spectral parameters (peak energy $E_{\mathrm{p}}$, low and high energy indices, $\alpha$ and $\beta$, and their time derivatives) evaluated at pulse maximum. Then, using a Yonetoku-like relation we obtain a theoretical lag-luminosity relation that is confronted with data.

Results. We first apply our model to the initial pulse of GRB 130427A, for which high quality data are available, to check quantitatively whether the measured lags are consistent with the observed spectral evolution. We then use a Monte Carlo approach to generate a sample of synthetic lags, which we compare to an observed sample of Swift bursts. The dispersion of both the observed and modelled lag-luminosity relations appears large, which questions the value of this relation as a reliable distance indicator.
\end{abstract}

Key words. gamma-ray burst: general - radiation mechanisms: non-thermal - distance scale

\section{Introduction}

It is well known that lags between gamma-ray burst (GRB) light curves observed in different spectral bands are a direct consequence of spectral evolution (Kocevski \& Liang 2003; Schaefer 2004; Ryde 2005; Hakkila \& Preece 2011). Obviously, with an invariant spectrum, all light curves would simply be proportional, therefore yielding zero lag. Lags provide an insight into the spectral evolution in GRBs from a temporal perspective. Moreover spectral lags have been found to correlate with burst luminosity and can be used as distance indicators (Norris et al. 2000; Norris 2002). In the present Swift and Fermi era more lags have been obtained in bursts with known distance, which allows the testing of the lag-luminosity relation with larger samples (Ukwatta et al. 2010, 2012; Bernardini et al. 2015; Heussaff 2015) or with better spectral coverage in individual events showing several pulses of different intensities (Zhang 2012; Shenoy et al. 2013).

Spectral evolution in GRBs generally goes from hard to soft within each pulse of the light curve. As a result, the pulse peaks earlier at high energy than at low energy, which corresponds (by definition) to a positive lag. Various mechanisms have been proposed to explain the spectral evolution, from a geometric origin resulting from the curvature of the emitting shells in the ejecta (Shen et al. 2005; Lu et al. 2006) to an intrinsic effect related to a true decrease of the peak energy with time (Kocevski \& Liang 2003; Daigne \& Mochkovitch 2003) or both (Peng et al. 2011).

To clarify the relationship between lags and spectral evolution Hafizi \& Mochkovitch (2007) and Boçi et al. (2010) developed a simple analytical approach where the lag for a given pulse in the light curve is obtained from a linear expansion of the spectral parameters at pulse maximum. It appears from their result that the lag depends on the full spectral evolution, including $E_{\mathrm{p}}$ and the spectral indices $\alpha$ and $\beta$ at low and high energy. In addition, the pulse shape and duration also affect the lag value for a given spectral evolution.

In the present work we use this approach to (i) analyse in some detail the lags measured with Fermi in the first pulse of the very bright burst GRB 130427A (Preece et al. 2014) and (ii) to statistically study a sample of lags obtained from the analysis of 70 Swift bursts (49 with known peak energy and luminosity). In Sect. 2 we present our analytical model and summarize its main results; the model is confronted with data in Sect. 3 and Sect. 4 is our conclusion.

\section{A simple theory of lags}

\subsection{Analytical approach}

The result obtained by Hafizi \& Mochkovitch (2007) can be summarized by the following expression:

$\delta t_{12}=\frac{f_{12, E_{\mathrm{p}}} \dot{e}_{\mathrm{p}}+f_{12, \alpha} \dot{a}+f_{12, \beta} \dot{b}}{C_{1}}$,

where the lag $\delta t_{12}$ between two energy bands $1\left[E_{1, \min }, E_{1, \max }\right]$ and $2\left[E_{2, \min }, E_{2, \max }\right]$ is defined as the difference in time between the pulse maxima in the two bands. This relation clearly establishes the link between lags and spectral evolution through 


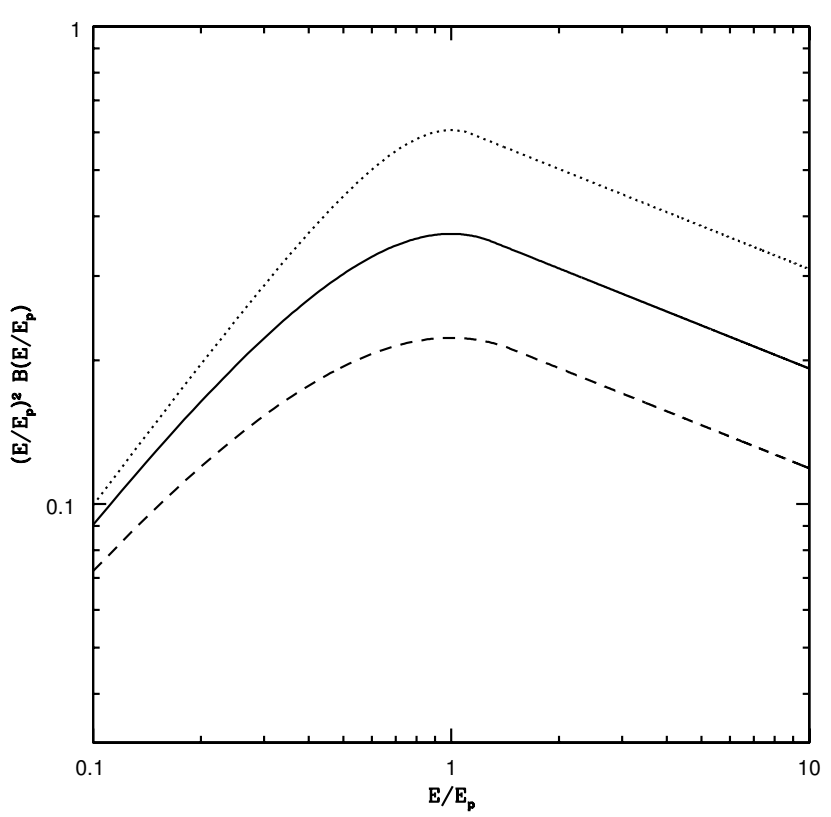

Fig. 1. $x^{2} \mathcal{B}(x)$ spectra (with $x=E / E_{\mathrm{p}}$ ) for $\alpha=-1, \beta=-2.3$ and $n=1$ in Eq. (4) (original Band function; full line), $n=2$ (dotted line), and $n=0.67$ (dashed line).

the three quantities $\dot{e}_{\mathrm{p}}, \dot{a}$, and $\dot{b}$, which are the logarithmic time derivatives of the peak energy $E_{\mathrm{p}}$, and low and high energy spectral indices $\alpha$ and $\beta$, evaluated at pulse maximum $t_{1}$ in the low energy band 1 . The three functions $f_{12, \mathrm{X}}$ in Eq. (1) are defined by

$f_{12, \mathrm{X}}=\left.\frac{\partial \log \mathcal{F}_{12}}{\partial \log X}\right|_{t_{1}}$,

where

$\mathcal{F}_{12}\left(E_{\mathrm{p}}, \alpha, \beta\right)=\frac{\int_{E_{2, \text { min }} / E_{\mathrm{p}}}^{E_{2, \max } / E_{\mathrm{p}}} \mathcal{B}_{\alpha \beta}(x) \mathrm{d} x}{\int_{E_{1, \text { min }} / E_{\mathrm{p}}}^{E_{1, \max } / E_{\mathrm{p}}} \mathcal{B}_{\alpha \beta}(x) \mathrm{d} x}$,

and $\mathcal{B}_{\alpha \beta}(x)$ is the spectral shape ${ }^{1}$, which we approximate by a modified Band function

$\mathcal{B}(x)=\left\{\begin{array}{l}x^{\alpha} \exp \left[-(\alpha+2) \frac{x^{n}}{n}\right] \text { for } x \leq x_{\mathrm{L}}=\left(\frac{\alpha-\beta}{\alpha+2}\right)^{1 / n} \\ x^{\beta} x_{\mathrm{L}}^{\alpha-\beta} \exp \left(\frac{\beta-\alpha}{n}\right) \text { otherwise. }\end{array}\right.$

The case with $n=1$ represents the original Band function while $n>1$ (resp. $n<1$ ) corresponds to a narrower (resp. broader) transition between the two power laws as shown in Fig. 1, in which we represented $x^{2} \mathcal{B}(x)$ for $n=1,2$ and 0.67 .

Finally, the $C_{1}$ parameter in Eq. (1) is the curvature of the pulse at time $t_{1}$

$C_{1}=\frac{\ddot{N}_{1}\left(t_{1}\right)}{N_{1}\left(t_{1}\right)}$,

where $N_{1}(t)$ is the photon flux in band 1 .

We can rewrite Eq. (1) in dimensionless form by introducing the pulse duration $t_{\mathrm{p}}$,

$\frac{\delta t_{12}}{t_{\mathrm{p}}}=\frac{f_{12, E_{\mathrm{p}}} \delta e_{\mathrm{p}}+f_{12, \alpha} \delta a+f_{12, \beta} \delta b}{\mathscr{C}_{1}}$,

1 We note that $\mathcal{F}_{12}$ is just the hardness ratio between the two spectral bands. where $\delta x=\dot{x} \times t_{\mathrm{p}}$ and $\mathscr{C}_{1}=C_{1} \times t_{\mathrm{p}}^{2}$. Equation (6) then shows that, for a given spectral evolution over the duration of the pulse (represented by the $\delta x$ values), the lag is proportional to the pulse duration and inversely proportional to the "spikiness" $\mathscr{C}_{1}$. Equation (6) therefore provides a simple explanation for the fact that short bursts generally have small lags.

\subsection{Lags as a function of energy}

\subsubsection{Fixed $E_{\mathrm{p}}$, different energy bands}

We first consider a pulse with a Band spectrum of peak energy $E_{\mathrm{p}}$, spectral indices, $\alpha$ and $\beta$, and temporal properties represented by the ratio $t_{\mathrm{p}} / \mathscr{C}$ of the pulse duration to the curvature at pulse maximum (in a low energy band [10-20] keV). We compute the lag between this band and another band covering the interval $[E-1.25 E]$, where $E$ is varied from $15 \mathrm{keV}$ to $10 \mathrm{MeV}$. We adopt $E_{\mathrm{p}}=500 \mathrm{keV}, \alpha=-1, \beta=-2.3$, and $t_{\mathrm{p}} / \mathscr{C}=0.3 \mathrm{~s}$, and we consider three cases of spectral evolution with $\delta e_{\mathrm{p}}=-0.5$ and $\delta a=\delta b=0,0.1$, and 0.2 . The results are shown in Fig. 2a. The low energy band is always in the $\alpha$ part of the spectrum so that a change of behaviour takes place when the high energy band crosses $E_{\mathrm{p}}$. The evolution of $\alpha$ (resp. $\beta$ ) mainly affects the lag below (resp. above) the break at $E \approx E_{\mathrm{p}}$. For a fixed spectral evolution, $\delta e_{\mathrm{p}}=-0.5$ and $\delta a=\delta b=0.1$, we also show in Fig. $2 \mathrm{~b}$ the results for a modified Band spectrum with $n=2$ and $n=0.67$ in Eq. (4) and for the case of a power law with an exponential cut-off.

\subsubsection{Fixed energy bands, different $E_{\mathrm{p}}$}

We now fix the two energy bands, 1 [100-150] keV and 2 [200$250] \mathrm{keV}$, which were used to obtain the lags in the source frame by Ukwatta et al. (2012), Bernardini et al. (2015), and Heussaff (2015), and in Fig. 3a we represent the lag as a function of the peak energy of the pulse spectrum. We first adopt a Band spectrum with $\alpha=-1, \beta=-2.3$ and $t_{\mathrm{p}} / \mathscr{C}=0.3 \mathrm{~s}$ and consider the same three cases of spectral evolution, $\delta e_{\mathrm{p}}=-0.5$, $\delta a=\delta b=0,0.1$ and 0.2 . As expected the lag is maximum when the peak energy lies just between the two bands, at about $E_{\mathrm{p}, *}=175 \mathrm{keV}$. If the spectral evolution is limited to the peak energy (i.e. $\delta a=\delta b=0$ ) the lag vanishes at low or high $E_{\mathrm{p}}$ values when the two energy bands are both in the same power-law part of the spectrum. Then the hardness ratio $\mathcal{F}_{12}\left(E_{\mathrm{p}}, \alpha, \beta\right)$ does not depend on $E_{\mathrm{p}}$ any more (see Eq. (3)) so that $f_{12, E_{\mathrm{p}}}=0$, leading to a zero lag if $\alpha$ and $\beta$ do not evolve. Conversely, if the spectral evolution also affects the spectral indices (which is generally the case) the lag at low (resp. high) $E_{\mathrm{p}}$ is fixed by the evolution of $\beta$ (resp. $\alpha$ ). Then in Fig. 3b we show, for $\delta e_{\mathrm{p}}=-0.5$, $\delta a=\delta b=0.1$, the results for a modified Band spectrum with $n=2$ or $n=0.67$ and for a power law with an exponential cut-off. In this last case, the lag steadily rises at low $E_{\mathrm{p}}$. This runaway feature however corresponds to the situation in which the flux in the high energy band shrinks owing to the exponential cut-off in the spectrum, which in practice would make the lag measurement very difficult.

\subsection{Comparison to a pulse model}

To check the validity of the previous first order derivation of the spectral lag, we constructed a simple pulse model with a typical 
R. Mochkovitch et al:: A simple theory of lags in GRBs
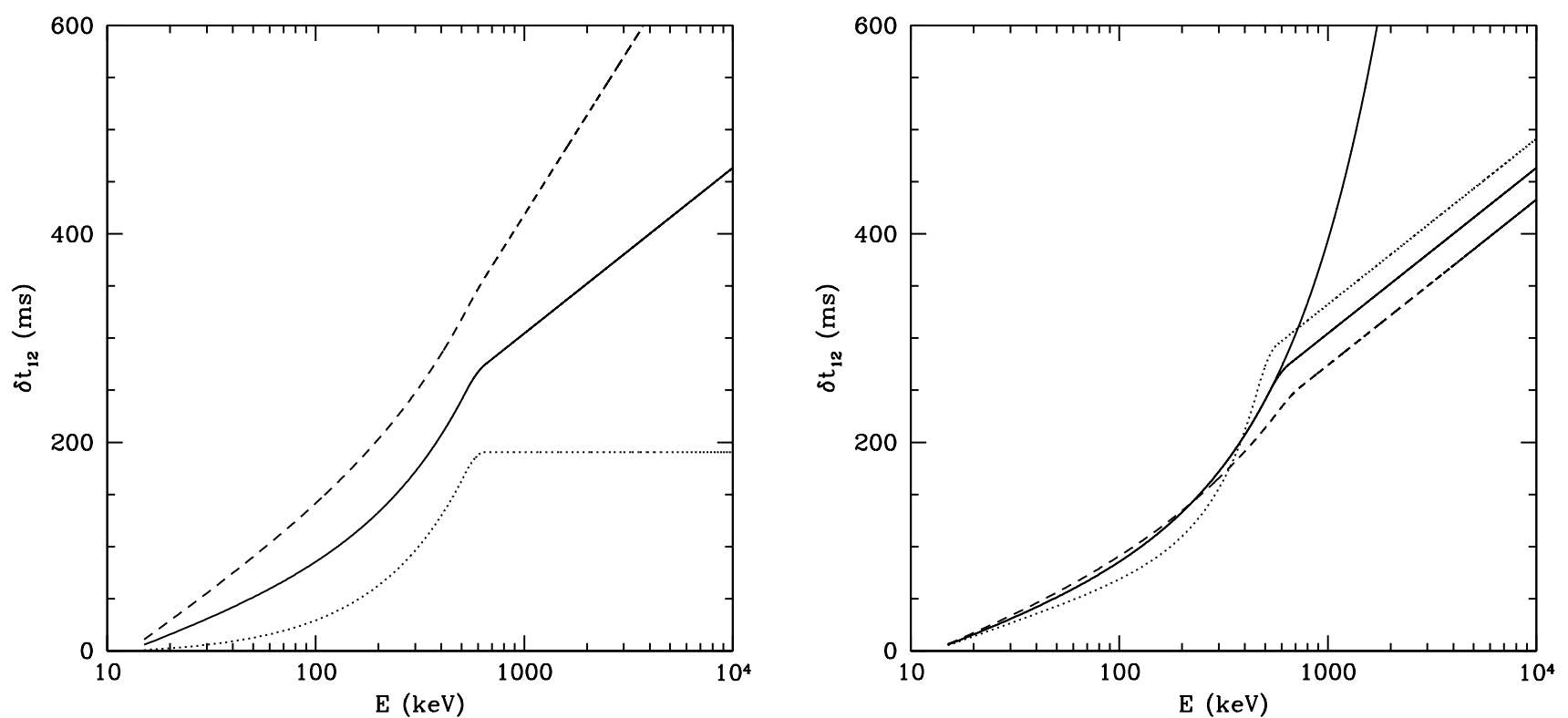

Fig. 2. a) Left panel: lags between the low energy band [10-20] keV and a moving band $[E-1.25 E]$ as a function of $E$ (in keV) for a pulse with $t_{\mathrm{p}} / \mathscr{C}=0.3 \mathrm{~s}$ and a Band spectrum $\left(n=1\right.$ in Eq. (4)) with $E_{\mathrm{p}}=500 \mathrm{keV}, \alpha=-1, \beta=-2.3$ for three different cases of spectral evolution: $\delta e_{\mathrm{p}}=-0.5$ and $\delta a=\delta b=0$ (dotted line), 0.1 (full line), and 0.2 (dashed line); b) right panel: same as a) for the case $\delta e_{\mathrm{p}}=-0.5$ and $\delta a=\delta b=0.1$ (full line), but also with a modified Band spectrum with $n=2$ (dotted line) and $n=0.67$ (dashed line). The full line with no break at $500 \mathrm{keV}$ corresponds to a power-law spectrum with $\alpha=-1$ and an exponential cut-off.
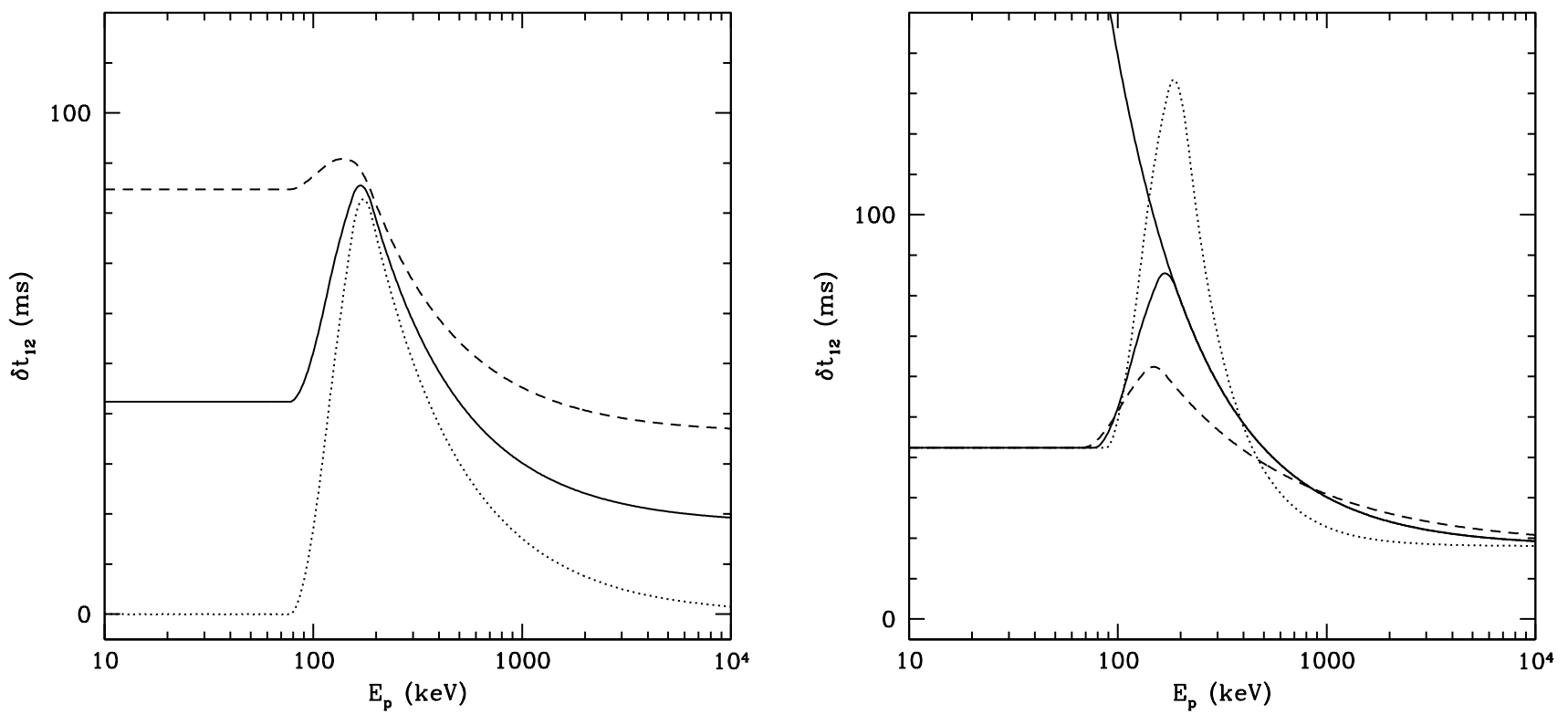

Fig. 3. a) Left panel: lags between the two bands, [100-150] and [200-250] keV, as a function of the peak energy of the pulse spectrum. The spectral indices at low and high energy are $\alpha=-1$ and $\beta=-2.3$. The curves correspond to the same three cases of spectral evolution considered in Fig. 2; b) right panel: same as a) for the case $\delta e_{\mathrm{p}}=-0.5$ and $\delta a=\delta b=0.1$ (full line), but also with a modified Band spectrum with $n=2$ (dotted line) and $n=0.67$ (dashed line). The full line that continuously rises at low $E_{\mathrm{p}}$ corresponds to a power-law spectrum with $\alpha=-1$ and an exponential cut-off.

fast rise and an exponential decay luminosity profile

$L(t)=L_{0} \times\left\{\begin{array}{l}2 x-x^{2} \text { for } x=\frac{t}{t_{\mathrm{p}}} \leq 1.5 \\ 0.75 \exp (-4 / 3 x+2) \text { for } x>1.5,\end{array}\right.$

so that the luminosity and its first derivative are continuous at $x=1.5$ and where the spectral evolution takes the form

$\left\{\begin{array}{l}E_{\mathrm{p}}=E_{\mathrm{p}, 0} \times \exp \left(\delta e_{\mathrm{p}} x\right) \\ \alpha=\alpha_{0} \times \exp (\delta a x) \\ \beta=\beta_{0} \times \exp (\delta b x),\end{array}\right.$ where $\delta e_{\mathrm{p}}, \delta a$, and $\delta b$ have been defined in Eq. (6). From Eqs. (7) and (8) and assuming a Band spectrum $(n=1$ in Eq. (4)), it is possible to compute the pulse profile in any spectral band. The results are shown in Fig. 4 for $E_{\mathrm{p}, 0}=1000 \mathrm{keV}, \alpha_{0}=-1, \beta_{0}=$ $-2.3, \delta e_{\mathrm{p}}=-0.5$, and $\delta a=\delta b=0.1$, and the two bands 1 [10$20]$ and 2 [100-125] keV. The spectral lag $\delta t_{12}$ is calculated as the time difference between the pulse maxima at $t_{\mathrm{max}, 1}=1.34 t_{\mathrm{p}}$ and $t_{\max , 2}=1.22 t_{\mathrm{p}}$, so that $\delta t_{12}=0.12 t_{\mathrm{p}}$.

We used this pulse model to check the analytical results. A comparison is shown in Fig. 5 for the lag-energy relation. As the 


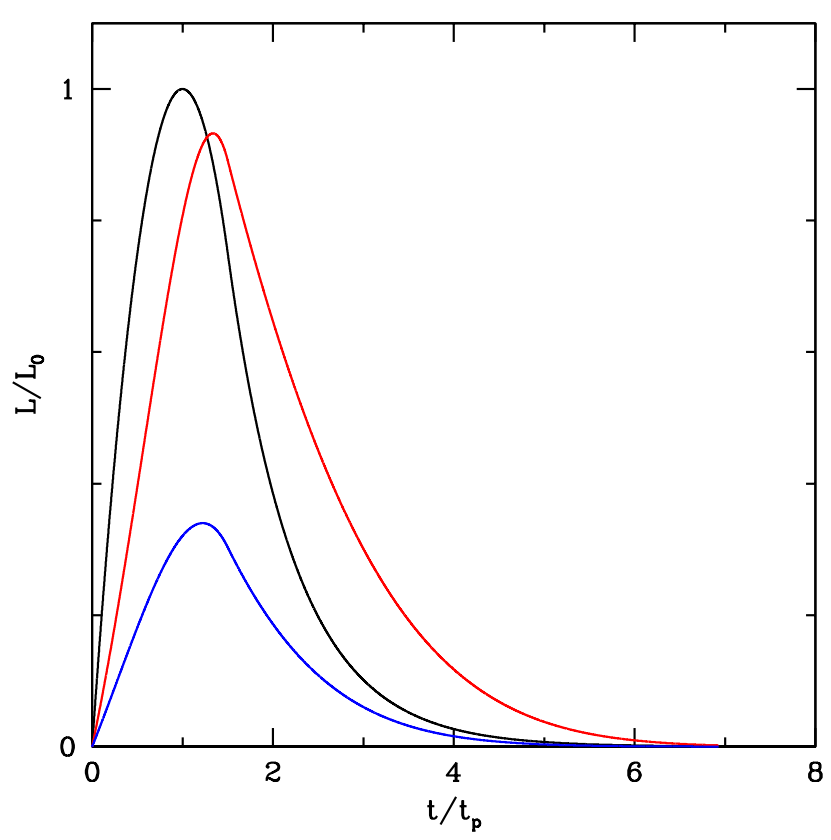

Fig. 4. Light curves for the pulse model. The black curve shows the luminosity while the red and blue curves represent the photon flux in the [10-20] and [100-125] keV range, respectively. The flux unit is arbitrary, but the flux ratio between the two bands is respected.

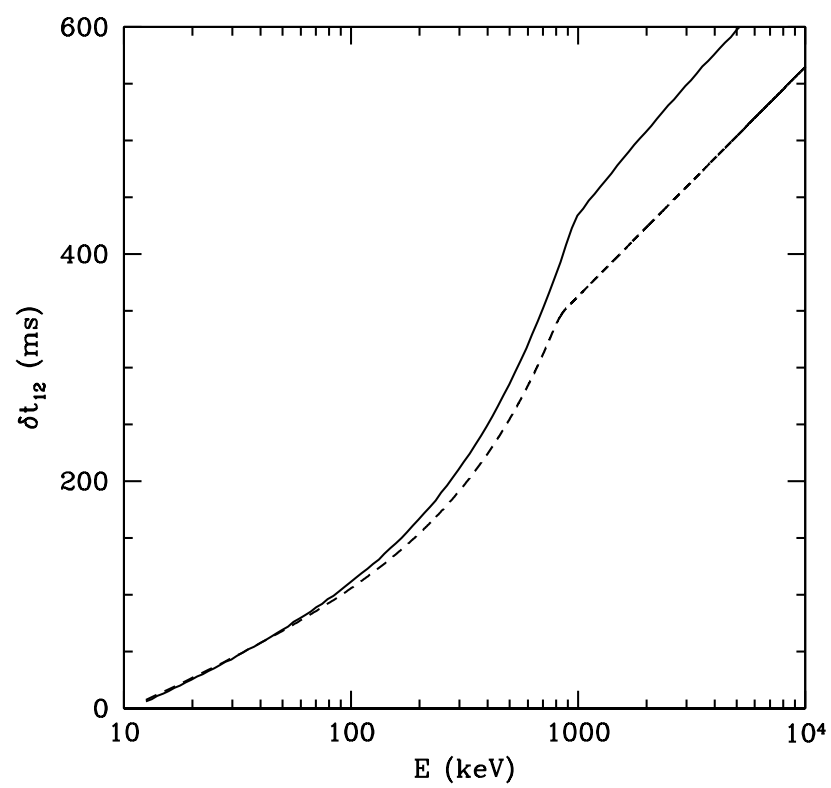

Fig. 5. Lags between the low energy band [10-20] keV and a moving band [ $E-1.25 E]$ as a function of $E$ (in $\mathrm{keV}$ ); comparison of the result from the pulse model (full line) and analytical expression (Eq. (1); dashed line).

analytical expression of the lag is obtained from a linear expansion around pulse maximum in the low energy band, we use in Eq. (6) the values of $E_{\mathrm{p}}, \alpha$, and $\beta$ at $t=t_{\max , 1}=1.34 \mathrm{~s}$ (i.e. assuming $t_{\mathrm{p}}=1 \mathrm{~s}$ ), which gives $E_{\mathrm{p}}=512 \mathrm{keV}, \alpha=-1.14$ and $\beta=-2.63$. The curvature is computed from the pulse model. We obtain $\left|\mathscr{C}_{1}\right|=3.1$, which is somewhat larger than the curvature of the luminosity light curve $\left|\mathscr{C}_{\mathrm{L}}\right|=2$, resulting from Eq. (7). It can be seen from Fig. 5 that the accuracy of the analytical expression is satisfactory, as the error does not exceed $20 \%$, even at the large energy difference between the two spectral bands.

\subsection{The lag-luminosity relation}

From the relation between the lag and peak energy at pulse maximum shown in Fig. 3, it is possible to obtain a lag-luminosity relation (LLR) if $E_{\mathrm{p}}$ is linked to the luminosity by a Yonetokulike relation (Yonetoku et al. 2004) of the form

$E_{\mathrm{p}}=E_{0} L_{52}^{\epsilon}$.

Adopting $E_{0}=300 \mathrm{keV}$ and $\epsilon=0.5$, we can directly transform Fig. 3a into the LLR shown in Fig. 6a. The LLR has two branches at low $\left(L<L_{*}\right)$ and high $\left(L>L_{*}\right)$ luminosity, where $L_{*} \sim 3.4 \times 10^{51} \mathrm{erg}$ is the luminosity corresponding to $E_{\mathrm{p}}=E_{\mathrm{p}, *}=175 \mathrm{keV}$. If the spectral indices do not vary $(\delta a=\delta b=0)$, the lag in the low luminosity branch vanishes when the two considered spectral bands both lie above $E_{\mathrm{p}}$. Then, the Band function simply reduces to a power law and $f_{12, E_{\mathrm{p}}}=0$. If $\delta b \neq 0$, the lag takes the fixed value

$\delta t_{12}=\frac{f_{12, \beta} \delta b}{\mathscr{C}_{1}} t_{\mathrm{p}}$

The situation is different when the spectrum is a power law with an exponential cut-off. In this case, the lag at low luminosity continuously increases. However (as already mentioned in Sect. 2.2.2 above) the flux in the high energy Band then becomes very low, making the lag measurement very difficult in practice.

In the high luminosity branch (i.e. at large $E_{\mathrm{p}}$ ), the function $f_{12, E_{\mathrm{p}}}$ behaves as $E_{\mathrm{p}}^{-1}$ so that, if $\delta a=0$, we get

$\delta t_{12} \propto L^{-\epsilon}$.

If $\delta a>0$ the LLR is steeper, becoming quasi-vertical when $\delta a>$ 0.2 . Finally, the variation of the LLR when the curvature of the spectrum is changed is shown in Fig. $6 \mathrm{~b}$ for $\delta e_{\mathrm{p}}=-0.5, \delta a=$ $\delta b=0.1$, and three values of $n=1,0.67$ and 2 in Eq. (4).

\section{Comparison to observations}

\subsection{Lags of pulses and global lags}

A potential problem in the comparison between data and model predictions comes from the fact that in the case of a complex burst, the measured lag is generally obtained by cross correlation of the light curves in the two considered spectral bands, while our model focuses on the lag of a single pulse, which is obtained as the time difference between the maxima in the two bands.

We first checked that for a single pulse this simple approach gives results that are close (within 10\%) to those obtained by cross corelation. Then, in a complex burst with many pulses, where the global lag represents some average of the individual lags, we want to estimate the weight of each pulse in this average.

This can be carried out in a simple way when the pulses in the light curve do not overlap. Then for each pulse $i$ the crosscorrelation function of the profiles in two spectral bands 1 and 2 can be expanded around the maximum,

$C_{i}(\delta t) \approx C_{i}\left(\delta t_{12, i}\right)-N_{1, i} N_{2, i} \frac{\left(\delta t-\delta t_{12, i}\right)^{2}}{2 t_{\mathrm{p}, i}} \mathscr{C}_{i}$,

where $N_{1, i}$ and $N_{2, i}$ are the peak photon fluxes, $\delta t_{12, i}$ is the spectral lag (for which $C_{i}(\delta t)$ is maximum), $t_{\mathrm{p}, i}$ is the duration of the pulse, and $\mathscr{C}_{i}$, a dimensionless measure of the pulse curvature (see Eq. (6)). The cross correlation for a profile with several 

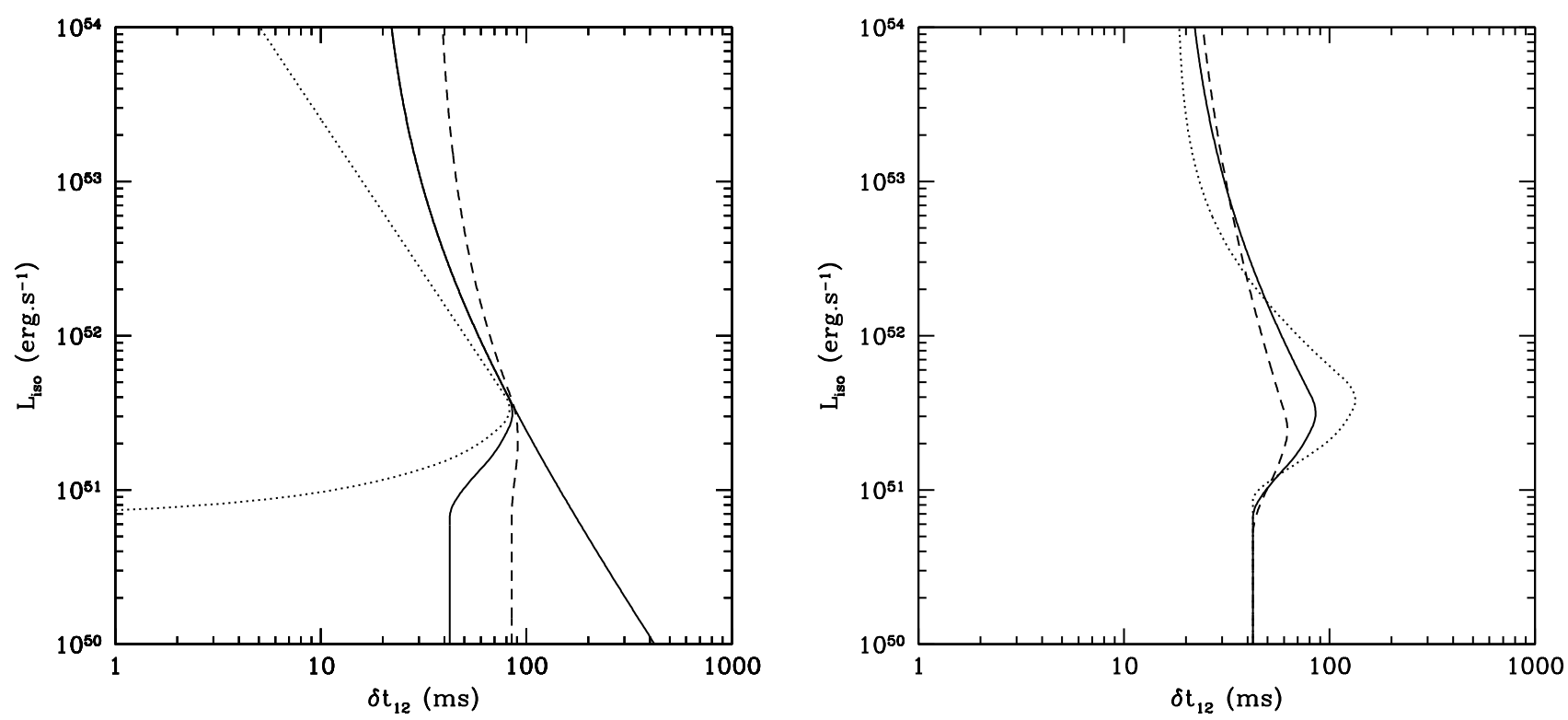

Fig. 6. a) Left panel: lag-luminosity relation for $\alpha=-1, \beta=-2.3, t_{\mathrm{p}} / \mathscr{C}=0.3 \mathrm{~s}, \delta e_{\mathrm{p}}=0.5$, and $\delta a=\delta b=0$ (dotted line), 0.1 (full line), and 0.2 (dashed line). The full line that extends to large lag values at low luminosity corresponds to a power-law spectrum with $\alpha=-1$ and an exponential cut-off; b) right panel: same as a) for the case $\delta e_{\mathrm{p}}=-0.5$ and $\delta a=\delta b=0.1$ (full line), but also with a modified Band spectrum with $n=2$ (dotted line) and $n=0.67$ (dashed line).

well-separated pulses can be approximated by the sum of the individual functions,

$C(\delta t) \approx \sum_{i} C_{i}(\delta t)$

The global lag $\delta t_{12}$ is then obtained by finding the maximum of $C(\delta t)$, yielding

$\delta t_{12}=\frac{\sum_{i} N_{1, i} N_{2, i} \mathscr{C}_{i} / t_{\mathrm{p}, i} \times \delta t_{12, i}}{\sum_{i} N_{1, i} N_{2, i} \mathscr{C}_{i} / t_{\mathrm{p}, i}}$

so that each individual lag appears in the average with a weight proportional to $N_{1, i} N_{2, i} \mathscr{C}_{i} / t_{\mathrm{p}, i}$. A bright, short or/and spiky pulse in a complex burst therefore imposes its lag on the whole event. In practice, the lag of brightest pulse generally dominates; see Hakkila et al. (2008). The global lag is averaged, however, in the case of several pulses with comparable weights, which can increase the dispersion of the LLR when it is generalized from individual pulses to the whole temporal history.

\subsection{Detailed study of spectral lags in individual bursts: GRB 130427A}

In some events, spectral lags have been obtained in several energy bands making possible a detailed comparison with the model and an estimate of the magnitude of spectral evolution. The best example is probably provided by the first pulse in GRB 130427A (Preece et al. 2014), where the high signal-tonoise ratio enabled a lag analysis from a few tens of $\mathrm{keV}$ to several MeV. Table 1 summarizes the results (transported in the burst rest frame). For the six lag value in Table 1 we also give the corresponding functions $f_{12, \mathrm{X}}$ that appear in Eq. $(6)^{2}$, where 1 represents the low energy band centred at $30 \mathrm{keV}$ and 2 represents the high energy band (from $100 \mathrm{keV}$ to $5 \mathrm{MeV}$ ). From Preece et al. (2014) we get the spectral parameters at pulse maximum in the low energy band (at $t_{\mathrm{obs}}=0.5 \mathrm{~s}$ ): $E_{\mathrm{p}}=650 \mathrm{keV}$

\footnotetext{
2 A pure Band spectrum was assumed in this analysis.
}

Table 1. Spectral lags (in burst rest frame) between a low energy band centred at $30 \mathrm{keV}$ and six other spectral bands for the first pulse in GRB 130427A; the corresponding functions $f_{12, \mathrm{X}}$ evaluated at pulse maximum in the low energy band are also given.

\begin{tabular}{ccccc}
\hline \hline Spectral bands & Lag $(\mathrm{s})$ & $f_{12, E_{\mathrm{p}}}^{i}$ & $f_{12, \alpha}^{i}$ & $f_{12, \beta}^{i}$ \\
\hline $30 \mathrm{keV}-100 \mathrm{keV}$ & 0.05 & 0.22 & -0.68 & 0 \\
$30 \mathrm{keV}-160 \mathrm{keV}$ & 0.10 & 0.36 & -0.90 & 0 \\
$30 \mathrm{keV}-400 \mathrm{keV}$ & 0.16 & 1. & -1.15 & 0 \\
$30 \mathrm{keV}-950 \mathrm{keV}$ & 0.22 & 2.27 & -1.09 & -1.1 \\
$30 \mathrm{keV}-1600 \mathrm{keV}$ & 0.28 & 2.36 & -1.08 & -2.3 \\
$30 \mathrm{keV}-4800 \mathrm{keV}$ & 0.35 & 2.36 & -1.08 & -4.9 \\
\hline
\end{tabular}

(rest frame), $\alpha=-0.57$, and $\beta=-3$; the value $\beta$ is not available for the first pulse and the adopted value corresponds to the whole event. We then obtain estimates of the three quantities, $x_{e}=\delta e_{\mathrm{p}} / Q, x_{a}=\delta a / Q$, and $x_{b}=\delta b / Q$, where $Q=\mathscr{C}_{1} / t_{\mathrm{p}}$, by minimizing the difference between the model results given by Eq. (6) and the observations

$\Delta=\sum_{1}^{N}\left[\delta t_{i}-\left(f_{12, \mathrm{E}_{\mathrm{p}}}^{i} x_{e}+f_{12, \alpha}^{i} x_{a}+f_{12, \beta}^{i} x_{b}\right)\right]^{2}$,

where the lags $\delta t_{i}$ and the functions $f_{12, \mathrm{X}}^{i}$ are given in Table 1. We began by considering only the first three lags $(N=3)$, which do not depend on $\beta$ and its evolution since the high energy spectral band is still in the $\alpha$ part of the Band function. These lags also have values that are much smaller than the pulse duration, which allows us to use the linear approach of Eq. (6). We then included all six lag values $(N=6)$, which is more constraining but also possibly less accurate because the lag for the highest energy band is not much smaller than the pulse duration and the adopted high energy index $\beta=-3$ is just an average over the whole burst duration. In the first case, the resulting values are $x_{e}=0.087$ and $x_{a}=-0.065$ ( $x_{b}$ is not constrained), while in the second we obtain $x_{e}=0.06, x_{a}=-0.08$ and $x_{b}=-0.025$, showing a difference of $35 \%$ in $x_{e}$ and of $20 \%$ in $x_{a}$. These values are then 
used to obtain the lag-energy relation, which is shown for the two cases in Fig. 7 together with the data points.

The time-resolved spectral analysis available for the first pulse partially allows us to test the consistency of the results. At pulse maximum one finds from the data that $\delta e_{\mathrm{p}} \simeq-1$ and $\delta a \simeq 0.6$. Then with $t_{\mathrm{p}}=0.5 \mathrm{~s} /(1+z) \approx 0.37 \mathrm{~s}$ (rest-frame value), we get $x_{e} \simeq-0.09\left(\frac{4}{\mathscr{C}_{1}}\right)$ and $x_{a} \simeq 0.055\left(\frac{4}{\mathscr{C}_{1}}\right)$. With $\mathscr{C}_{1}=4$, which seems reasonable even if a highly accurate measure of the curvature is difficult, the results obtained from the first three lags are in very good agreement with the observed spectral evolution. When all six lag values are included in the analysis, the agreement is less satisfactory, probably from the combined effects of using Eq. (6) out of its domain of validity and the uncertainty in $\beta$.

\subsection{Monte Carlo approach to the observed lag-luminosity relation}

Ukwatta et al. (2012), Bernardini et al. (2015), and Heussaff (2015) recently constructed samples of rest-frame lags for long bursts with known redshift observed with the Swift satellite. From the 49 (of 70) long GRBs in the Heussaff (2015) sample (see Table 2), which have a measured peak energy and luminosity, 20 have a positive lag while the error bars for the others only allow us to fix an upper limit to the lag (in one case, this upper limit is negative). For comparison with our analytical results, we developed a simple Monte Carlo approach, in which the spectral and temporal parameters of the generated events are drawn according to the following distributions ${ }^{3}$ :

- The low energy spectral index $\alpha$ has a normal distribution centred at $\alpha=-1$ with a standard deviation $\sigma_{\alpha}=0.25$ (Kaneko et al. 2006).

- The high energy spectral index $\beta$ has a truncated normal distribution centred at $\beta=-2.3$ with the same standard deviation but truncated to exclude all values of $\beta>-2$ (Kaneko et al. 2006).

- The pulse duration also follows a log-normal distribution with $\left\langle\log t_{\mathrm{p}}\right\rangle=0$ and $\sigma_{\text {Log } t_{\mathrm{p}}}=0.35$ (Nakar \& Piran 2002).

- The peak energy follows a log-normal distribution with $\left\langle\log E_{\mathrm{p}}\right\rangle=2.7$ (rest frame) and $\sigma_{\log E_{\mathrm{p}}}=0.25$ (Kaneko et al. 2006; Ghirlanda et al. 2012).

- The luminosity is obtained from the peak energy using the Yonetoku relation (Eq. (9)) where we added a dispersion of 0.5 in $\log L$.

The parameters controlling the spectral evolution, $\delta e_{\mathrm{p}}, \delta a$, and $\delta b$, and the spikiness $\mathscr{C}$, are weakly constrained. We first fix their values to $\delta e_{\mathrm{p}}=-0.5, \delta a=\delta b=0,0.1$, or 0.2 and $|\mathscr{C}|=3.33$ (so that $\left\langle t_{\mathrm{p}}\right\rangle /|\mathscr{C}| \simeq 0.3$. We then draw the burst parameters according to the above rules and get the corresponding lag using Eq. (6). The slope of the resulting LLR strongly depends on the adopted value for $\delta a$, going from -2.4 for $\delta a=0$ to -5 for $\delta a=0.1$ and -7.5 for $\delta a=0.2$. These slopes (especially for $\delta a \neq 0$ ) appear too steep when compared to the data. One possible way to solve this discrepancy is to introduce some kind of

These distributions, as well as the Yonetoku relation, are deduced from observed samples and therefore are not free from selection effects. Consequently the lag-luminosity relation obtained from our Monte Carlo simulations is not intrinsic as it includes these selection effects by construction. It should then be compared with data subject to similar selection effects.

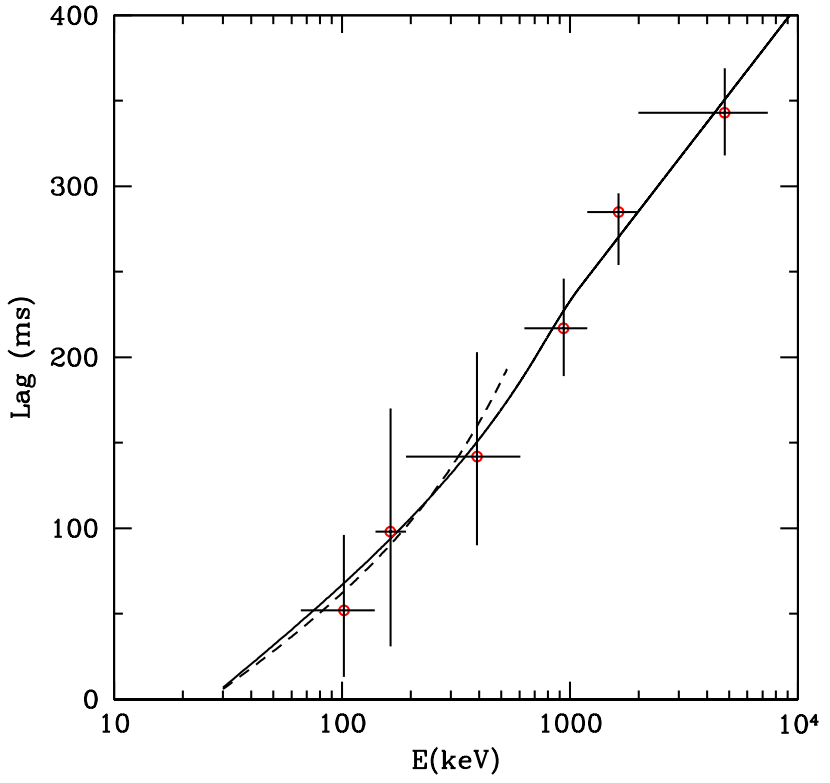

Fig. 7. Lags as a function of energy in GRB 130427A. The six measured values (Preece et al. 2014) are fitted using our model with $E_{\mathrm{p}}=$ $650 \mathrm{keV}, \alpha=-0.57, \beta=-3$ and $x_{e}=0.061, x_{a}=-0.08, x_{b}=-0.025$ (full line) and $x_{e}=0.087, x_{a}=0.065$ (dashed line); see text for details.

variability-luminosity relation of the form

$\frac{\left\langle t_{\mathrm{p}}\right\rangle}{|\mathscr{C}|}=0.3\left(\frac{L}{10^{52} \mathrm{erg} \mathrm{s}^{-1}}\right)^{\gamma}$,

with $\gamma \sim 0.1-0.2$, i.e. corresponding to a situation where the pulses become narrower or/and spikier as their luminosity increases. A few observational studies have suggested these relations (Reichart et al. 2001; Hakkila et al. 2008), but they would clearly need further confirmation. Adopting $\gamma=0.15$, the three slopes are reduced to $-0.4,-2.6$, and -3.1 for $\delta a=0,0.1$ and 0.2 , respectively. The case with $\delta a=0.1$ is shown in Fig. 8, where we represented the LLR together with the cumulative and differential distribution of lags. These lags are compared to the data from Heussaff (2015). It can be seen that the main difference comes from observed bursts with negative lags that are not produced by the simulation.

If we suppose that these negative lags result from measurement uncertainties and can be ignored, the model fits the data well. Conversely, if the existence of negative lags is confirmed (see Chen et al. 2005; Roychoudhury et al. 2014 for details) one simple way to produce them would be to suppose that the time derivative of the low energy spectral index $\alpha$ at pulse maximum can be positive (i.e. $\delta a$ negative in Eq. (6) corresponding to a soft to hard evolution for $\alpha$ ). For example, adopting for $\delta a$ a normal distribution centred at 0 with a dispersion of $0.15,15 \%$ of the lags are negative. The corresponding LLR and distribution of lags are shown in Fig. 9 (the negative lags do not appear in the LLR owing to the logarithmic scale) showing an excellent agreement with the data; a KS test indicates that the difference of 0.157 between the observed and predicted cumulative distribution functions has a probability of $17 \%$ of happening from random fluctuations. The above results were obtained with a Band spectrum ( $n=1$ in Eq. (4)) but we checked how they are modified when we adopt (i) a power law with an exponential cut-off or (ii) a value of $n$ different from unity. In the first case, the effect is moderate since, for a majority of the simulated spectra, the two considered spectral bands lie below $E_{\mathrm{p}}$ whose central value is $500 \mathrm{keV}$ (rest frame) in our simulation. In the second 
R. Mochkovitch et al.: A simple theory of lags in GRBs

Table 2. The 49 bursts with redshift and measured peak energy and luminosity in Heussaff (2015).

\begin{tabular}{|c|c|c|c|c|c|c|c|c|c|}
\hline GRB name & Redshift & $L_{\text {iso }}\left(10^{52} \mathrm{erg} \mathrm{s}^{-1}\right)$ & $E_{\mathrm{p}}(\mathrm{keV})$ & Lag (ms) & GRB name & Redshift & $L_{\text {iso }}\left(10^{52} \operatorname{erg~s}^{-1}\right)$ & $E_{\mathrm{p}}(\mathrm{keV})$ & Lag (ms) \\
\hline 140419A & 3.96 & $28.35 \pm 1.16$ & $1452_{-416}^{+416}$ & $13 \pm 37$ & 090424 & 0.54 & $1.47 \pm 0.04$ & $237_{-6}^{+6}$ & $29 \pm 16$ \\
\hline $140304 \mathrm{~A}$ & 5.28 & $8.92 \pm 1.05$ & $775_{-173}^{+173}$ & $28 \pm 48$ & 090423 & 8.00 & $16.04 \pm 1.89$ & $614_{-155}^{+155}$ & $11 \pm 57$ \\
\hline $140213 \mathrm{~A}$ & 1.21 & $3.31 \pm 0.11$ & $191_{-8}^{+8}$ & $-40 \pm 31$ & 090102 & 1.55 & $2.98 \pm 0.43$ & $1074_{-46}^{+46}$ & $-27 \pm 414$ \\
\hline 131030A & 1.29 & $5.72 \pm 0.14$ & $406_{-23}^{+23}$ & $36 \pm 51$ & 081222 & 2.77 & $10.13 \pm 0.26$ & $538_{-36}^{+36}$ & $39 \pm 39$ \\
\hline 130907A & 1.24 & $9.65 \pm 0.19$ & $882_{-25}^{+25}$ & $7 \pm 3$ & 080721 & 2.60 & $52.31 \pm 4.51$ & $1747_{-213}^{+241}$ & $-21 \pm 58$ \\
\hline $130701 \mathrm{~A}$ & 1.16 & $2.12 \pm 0.09$ & $192_{-9}^{+9}$ & $-8 \pm 32$ & 080607 & 3.04 & $64.50 \pm 3.07$ & $1590_{-133}^{+141}$ & $2 \pm 14$ \\
\hline 130427A & 0.34 & $11.57 \pm 0.16$ & $1112_{-7}^{+7}$ & $9 \pm 10$ & 080605 & 1.64 & $8.53 \pm 0.26$ & $768_{-198}^{+198}$ & $21 \pm 10$ \\
\hline $121128 \mathrm{~A}$ & 2.20 & $4.83 \pm 0.15$ & $244_{-10}^{+10}$ & $1 \pm 3$ & 080413B & 1.10 & $1.51 \pm 0.06$ & $141_{-17}^{+27}$ & $71 \pm 31$ \\
\hline $120811 C$ & 2.67 & $2.44 \pm 0.12$ & $204_{-20}^{+20}$ & $107 \pm 115$ & 080413A & 2.43 & $5.38 \pm 0.19$ & $433_{-144}^{+450}$ & $-5 \pm 15$ \\
\hline 120119A & 1.73 & $4.87 \pm 0.14$ & $500_{-22}^{+22}$ & $-1 \pm 18$ & 080411 & 1.03 & $5.59 \pm 0.12$ & $526_{-55}^{+71}$ & $24 \pm 12$ \\
\hline $110731 \mathrm{~A}$ & 2.83 & $25.49 \pm 0.70$ & $1223_{-75}^{+75}$ & $4 \pm 7$ & 080319C & 1.95 & $6.03 \pm 0.35$ & $906_{-271}^{+416}$ & $91 \pm 55$ \\
\hline $110715 \mathrm{~A}$ & 0.82 & $2.68 \pm 0.05$ & $218_{-20}^{+22}$ & $33 \pm 10$ & 080319B & 0.94 & $6.90 \pm 0.14$ & $1261_{-25}^{+27}$ & $7 \pm 3$ \\
\hline $110503 \mathrm{~A}$ & 1.61 & $0.53 \pm 0.02$ & $572_{-50}^{+52}$ & $28 \pm 42$ & 071117 & 1.33 & $2.77 \pm 0.10$ & $648_{-317}^{+317}$ & $142 \pm 39$ \\
\hline $110422 \mathrm{~A}$ & 1.77 & $12.06 \pm 0.39$ & $421_{-14}^{+14}$ & $43 \pm 31$ & 071020 & 2.14 & $15.81 \pm 0.56$ & $1014_{-167}^{+252}$ & $9 \pm 3$ \\
\hline $110205 \mathrm{~A}$ & 2.22 & $2.83 \pm 0.16$ & $715_{-238}^{+238}$ & $18 \pm 101$ & 071010B & 0.95 & $0.49 \pm 0.02$ & $88_{-14}^{+8}$ & $193 \pm 116$ \\
\hline 100906A & 1.73 & $4.56 \pm 0.18$ & $289_{-55}^{+48}$ & $37 \pm 71$ & 071003 & 1.10 & $3.03 \pm 0.19$ & $1678_{-210}^{+260}$ & $-3 \pm 72$ \\
\hline $100814 \mathrm{~A}$ & 1.44 & $0.72 \pm 0.06$ & $331_{-26}^{+26}$ & $94 \pm 81$ & 070521 & 0.55 & $2.04 \pm 0.08$ & $522_{-56}^{+56}$ & $19 \pm 33$ \\
\hline $100728 \mathrm{~B}$ & 2.11 & $2.90 \pm 0.41$ & $\begin{array}{l}341_{-68}^{+68} \\
\end{array}$ & $21 \pm 171$ & 061121 & 1.31 & $8.04 \pm 0.18$ & $1402_{-167}^{+208}$ & $13 \pm 5$ \\
\hline $100621 \mathrm{~A}$ & 0.54 & $0.25 \pm 0.01$ & $146_{-20}^{+28}$ & $436 \pm 363$ & 061007 & 1.26 & $6.23 \pm 0.16$ & $1065_{-81}^{+81}$ & $37 \pm 14$ \\
\hline 091208B & 1.06 & $1.67 \pm 0.11$ & $255_{-40}^{+41}$ & $-16 \pm 46$ & 060814 & 0.84 & $4.37 \pm 0.17$ & $750_{-169}^{+356}$ & $113 \pm 194$ \\
\hline 091127 & 0.49 & $0.65 \pm 0.04$ & $53_{-2}^{+2}$ & $86 \pm 235$ & 060206 & 4.04 & $6.44 \pm 0.39$ & $379_{-61}^{+61}$ & $53 \pm 21$ \\
\hline 091029 & 2.75 & $1.71 \pm 0.10$ & $230_{-94}^{+34}$ & $-7 \pm 48$ & 050922C & 2.20 & $5.35 \pm 0.24$ & $417_{-86}^{+163}$ & $-4 \pm 34$ \\
\hline 090812 & 2.45 & $8.27 \pm 0.46$ & $2023_{-525}^{+839}$ & $41 \pm 37$ & $050525 \mathrm{~A}$ & 0.61 & $0.75 \pm 0.02$ & $135_{-3}^{+3}$ & $35 \pm 9$ \\
\hline 090715B & 3.00 & $5.50 \pm 0.29$ & $536_{-120}^{+224}$ & $14 \pm 98$ & 050401 & 2.90 & $12.34 \pm 1.06$ & $515_{-62}^{+62}$ & $66 \pm 27$ \\
\hline 090618 & 0.54 & $1.02 \pm 0.02$ & $226_{-6}^{+6}$ & $4 \pm 119$ & & & & & \\
\hline
\end{tabular}
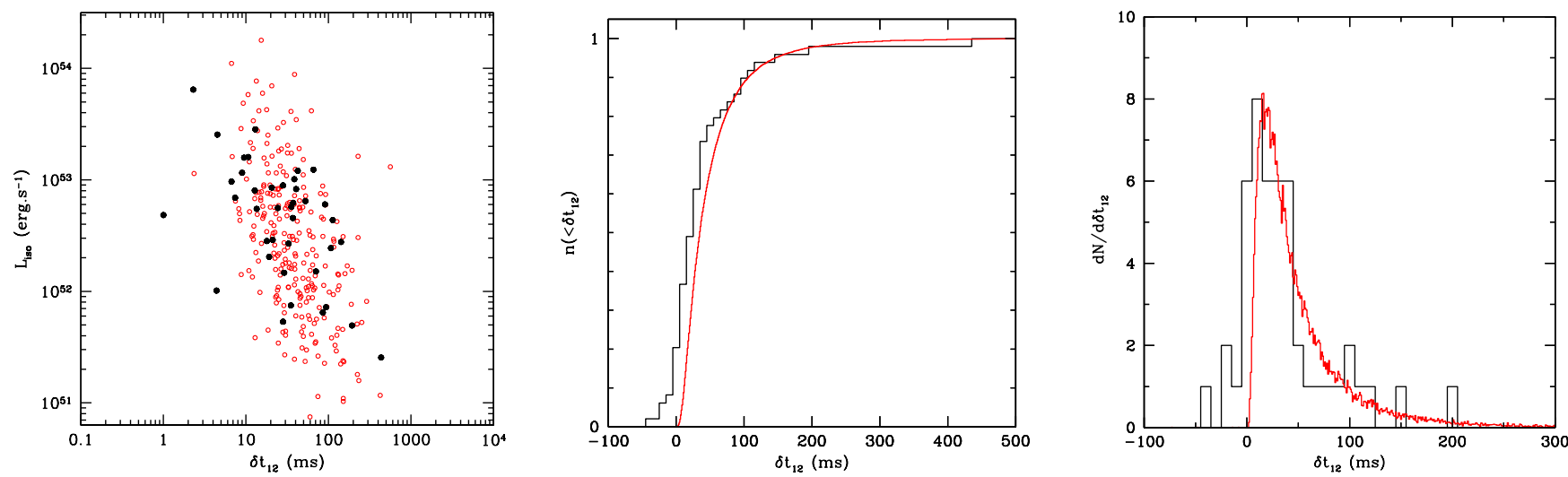

Fig. 8. Lag-luminosity relation, cumulative, and differential distributions of lags for $\delta e_{\mathrm{p}}=0.5, \delta a=\delta b=0.1$, and $\left\langle t_{\mathrm{p}}\right\rangle /|\mathscr{C}|$ given by Eq. (15) with $\gamma=0.15$; left panel: 250 simulated events (red circles) and data points from Heussaff (2015; black dots); middle panel: cumulative distribution of lags; right panel: differential distribution of lags. In the middle and right panels the observed distribution is binned in interval of $10 \mathrm{~ms}$.

case, we tested the value $n=2$, corresponding to an increased curvature at the peak. It allows us to obtain the same slopes for the LLR with $\gamma=0.05$ only, i.e. practically without supposing the variability-luminosity relation of Eq. (15).

\section{Conclusions}

Based on our previous work (Hafizi \& Mochkovitch 2007; Boçi et al. 2010), we compared the results from a simple analytical expression of the spectral lags in GRBs to data collected by the Swift and Fermi satellites. This expression (Eq. (6)) explicitly connects the lag of a pulse in the light curve to its spectral ( $E_{\mathrm{p}}, \alpha, \beta$, and their time derivatives) and temporal (pulse duration and curvature at the peak) parameters. We checked the accuracy of the analytical expression with a pulse model. It proved to be accurate within $20 \%$ even when the two considered spectral bands are wide apart. For the two bands [100-150] and [200$250] \mathrm{keV}$, we obtained the lag as a function of the peak energy of the spectrum (Fig. 3). Then, assuming a Yonetoku-like relation between the peak energy at pulse maximum and the luminosity, 

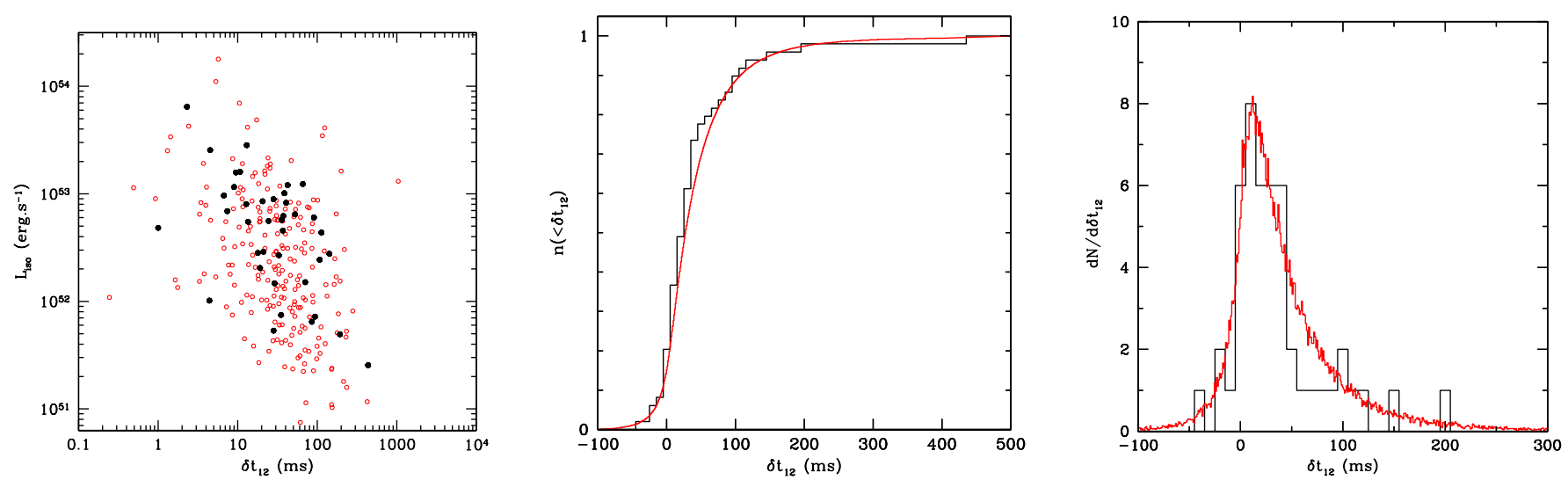

Fig. 9. Same as Fig. 8 but $\delta a$ now has a normal distribution with $\langle\delta a\rangle=0$ and a dispersion of 0.15 .

we derived a theoretical LLR (Fig. 6). When the spectral evolution is limited to variations of the peak energy ( $\alpha$ and $\beta$ staying constant) the lag is given by a power law, $\delta t \propto L^{-\epsilon}$ at high luminosity, where $\epsilon$ is the exponent of the Yonetoku-like relation $E_{\mathrm{p}} \propto L^{\epsilon}$. If the spectral indices also vary (from hard to soft within a pulse), the LLR becomes steeper than $L^{\epsilon}$.

We first tested the relation between lags and energy using Fermi data for the first pulse in the light curve of GRB 130427A. The fit of six lag values allows us to constrain the evolution of both $E_{\mathrm{p}}$ and the spectral indices, and the consistency of the results can be tested with the time-resolved spectral analysis available for this event. We then used a Monte Carlo approach where the spectral and temporal parameters were drawn according to the observed distributions and the results were compared to the lag analysis of 49 Swift bursts with known peak energy and luminosity performed by Heussaff (2015). The synthetic LLR appears steeper than the observed LLR, which can be corrected if the duration $t_{\mathrm{p}}$ or/and curvature $\mathscr{C}$ of the pulses are correlated to the luminosity. We presented results with a moderate correlation $\left\langle t_{\mathrm{p}}\right\rangle /|\mathscr{C}| \propto L^{0.15}$, which are in good agreement with the data except for the fact that the synthetic distribution does not show any negative lag contrary to the observed distribution. If these negative lags are real, a simple way to produce them in the model would be to allow the low energy spectral index to evolve from soft to hard in some cases.

Both the observational and model LLRs show that the lags globally decrease with increasing luminosity. However the correlation is rather steep and has a large dispersion, which probably prevents from using lags as an accurate distance indicator. In Fig. 8, with fixed values of $\delta e_{\mathrm{p}}, \delta a$, and $\delta b$, the typical luminosity interval for a given lag typically spans one order of magnitude already. With a dispersion added for $\delta a$ (Fig. 9, left panel) this interval rises to 1.5 orders of magnitude. At low luminosity (below $10^{51} \mathrm{erg} \mathrm{s}^{-1}$, where very limited data is available) a variety of behaviours are possible, as shown in Fig. 6. If the spectrum is a genuine or modified Band function, the lag vanishes if the high energy spectral index does not vary or takes a fixed value in the opposite (more realistic) case. If the spectrum is a power law with an exponential cut-off, the lag continuously increases but the low flux in the high energy band makes it difficult to measure in practice.

The comparison between our model and the data relied on a collection bursts mostly observed by Swift. As a result of the limited spectral coverage of the BAT instrument, the interval between the spectral bands used to obtain the lags is reduced, which results in small lag values that are easily affected by uncertainties. With Fermi and the future Chinese-French SVOM mission (Cordier et al. 2015) better lag estimates can and will be obtained using more separated spectral bands that will better constrain the spectral evolution, as illustrated here in the case of GRB 130427. In addition, SVOM will be able to provide the lags and the reshift simultaneously, which will allow for better testing of the LLR and its value as a distance indicator.

Acknowledgements. It is a pleasure to thank Frédéric Daigne for helpful discussions and the anonymous referee for valuable comments, which helped to improve the manuscript.

\section{References}

Bernardini, M. G., Ghirlanda, G., Campana, S., et al. 2015, MNRAS, 446, 1129 Boçi, S., Hafizi, M., \& Mochkovitch, R. 2010, A\&A, 519, A76

Chen, L., Lou, Y.-Q., Wu, M., et al. 2005, ApJ, 619, 983

Cordier, B., Wei, J., Atteia, J.-L., et al. 2015, ArXiv e-prints [arXiv: 1512.03323]

Daigne, F., \& Mochkovitch, R. 2003, MNRAS, 342, 587

Ghirlanda, G., Nava, L., Ghisellini, G., et al. 2012, MNRAS, 420, 483

Hafizi, M., \& Mochkovitch, R. 2007, A\&A, 465, 67

Hakkila, J., \& Preece, R. D. 2011, ApJ, 740, 104

Hakkila, J., Giblin, T. W., Norris, J. P., Fragile, P. C., \& Bonnell, J. T. 2008, ApJ, 677, L81

Heussaff, V. 2015, Ph.D. Thesis, Université Toulouse III, Paul Sabatier

Kaneko, Y., Preece, R. D., Briggs, M. S., et al. 2006, ApJS, 166, 298

Kocevski, D., \& Liang, E. 2003, ApJ, 594, 385

Lu, R.-J., Qin, Y.-P., Zhang, Z.-B., \& Yi, T.-F. 2006, MNRAS, 367, 275

Nakar, E., \& Piran, T. 2002, MNRAS, 331, 40

Norris, J. P. 2002, ApJ, 579, 386

Norris, J. P., Marani, G. F., \& Bonnell, J. T. 2000, ApJ, 534, 248

Peng, Z. Y., Yin, Y., Bi, X. W., Bao, Y. Y., \& Ma, L. 2011, Astron. Nachr., 332, 92

Preece, R., Burgess, J. M., von Kienlin, A., et al. 2014, Science, 343, 51

Reichart, D. E., Lamb, D. Q., Fenimore, E. E., et al. 2001, ApJ, 552, 57

Roychoudhury, A., Sarkar, S. K., \& Bhadra, A. 2014, ApJ, 782, 105

Ryde, F. 2005, A\&A, 429, 869

Schaefer, B. E. 2004, ApJ, 602, 306

Shen, R.-F., Song, L.-M., \& Li, Z. 2005, MNRAS, 362, 59

Shenoy, A., Sonbas, E., Dermer, C., et al. 2013, ApJ, 778, 3

Ukwatta, T. N., Stamatikos, M., Dhuga, K. S., et al. 2010, ApJ, 711, 1073

Ukwatta, T. N., Dhuga, K. S., Stamatikos, M., et al. 2012, MNRAS, 419, 614

Yonetoku, D., Murakami, T., Nakamura, T., et al. 2004, ApJ, 609, 935

Zhang, F.-W. 2012, Ap\&SS, 339, 123 